\title{
Endovascular therapy in Marfan syndrome: PRO
}

\author{
Bruce Tjaden $\mathrm{Jr}^{1}$, Ali Azizzadeh ${ }^{2}$ \\ ${ }^{1}$ Department of Cardiothoracic and Vascular Surgery, McGovern Medical School at the University of Texas Health Science Center at Houston \\ (UTHealth), Houston, Texas, USA; ${ }^{2}$ Division of Vascular Surgery, Cedars Sinai Medical Center, Los Angeles, CA, USA \\ Correspondence to: Bruce Tjaden Jr, MD. Department of Cardiothoracic and Vascular Surgery, McGovern Medical School at UTHealth, 6400 Fannin \\ Street, Suite 2850, Houston, Texas 77030, USA. Email: Bruce.L.Tjaden@uth.tmc.edu.
}

\begin{abstract}
Marfan syndrome (MFS) is an inherited connective tissue disorder that is associated with arterial fragility and aortic pathologies. The endovascular treatment of patients with MFS is controversial. Published series suggest that while endovascular intervention can be carried out with a low rate of immediate morbidity and mortality, midterm follow up demonstrates sizeable numbers of complications. However, in certain situations-such as rupture, reintervention for patch aneurysms, and elective interventions in which the landing zone is within a previously placed graft-endovascular interventions are appropriate. We present a review of the literature and our institutional perspective on this complex topic.
\end{abstract}

Keywords: Endovascular; Marfan; connective tissue; aorta

Submitted Jun 05, 2017. Accepted for publication Oct 23, 2017.

doi: 10.21037/acs.2017.10.06

View this article at: http://dx.doi.org/10.21037/acs.2017.10.06

\section{Introduction}

The advent of endovascular therapy has revolutionized the field of cardiovascular surgery, allowing for the treatment of a wide spectrum of pathologies while minimizing pain, recovery time, morbidity, and mortality. Patients with connective tissue disorders, such as Marfan syndrome (MFS), are prone to early development of aneurysms, dissections, and ruptures - and will often require numerous vascular interventions over their lifetimes. However, these patients are also prone to complications from percutaneous access and progressive degeneration of neighboring arterial segments, which has led to a hesitance to implement endovascular therapy in this population.

Historical publications have recommended against even diagnostic arteriography in patients with connective tissue disorders, due to a reported complication rate of nearly $70 \%$ (1). Furthermore, the radial force of an intraluminal stent or stent graft is perceived to put the landing zones at risk for hastened aneurysmal degeneration or deviceinduced dissection $(2,3)$. Despite these concerns (or perhaps accounting for them), endovascular stent grafting in these patients has never been formally studied (3). Increasing experience has dispelled some historic concerns and supported others. Overall, it is becoming obvious that endovascular therapy is a useful tool in the armamentarium of surgeons treating these patients. In fact, an endovascular or hybrid intervention may be the most suitable approach for patients with connective tissue disorders in some situations.

In this article, we will review the current body of evidence in support of endovascular treatment for arterial disease in patients with MFS and will also describe our institutional practices for these procedures.

\section{Historical treatment of arterial disease in MFS}

MFS was first described in the late 1800s by Dr. Antoine Bernard-Jean Marfan, a French pediatrician. However, it was not until a half century later that the aortic ramifications of the disease were noted. These include a predisposition to aneurysm development, dissection, and rupture-often of the root of the aorta (3-6). On a microscopic level, replacement of apoptotic aortic smooth muscle cells with mucinous debris is referred to as cystic medial necrosis $(3,5)$ and underlies the vessel fragility associated with the disease. Historically, patients with MFS had a significantly shortened life expectancy (3). 
Aortic root and ascending aortic reconstruction in patients with MFS have typically involved aortic valve resuspension and root replacement in elective cases, and composite mechanical valve + graft repair in emergencies (3). Descending thoracic aortic aneurysms (DTAA) and thoracoabdominal aortic aneurysms (TAAA) are carried out in much the same fashion as for patients without MFS, although the amount of aorta that requires replacement is often greater (3). Aneurysmal degeneration of "Carrel" inclusion patches (renovisceral or intercostal) is one of the most common indications for reintervention in these postoperative patients $(3,4)$. Open repair for patch aneurysm is associated with a high mortality $(40 \%$ in one series) $(4,7)$.

\section{Current evidence on endovascular therapy in MFS}

Several consensus recommendations exist to guide decisionmaking in MFS patients considered for endovascular repair. First, endovascular therapy has been recommended only if patients are felt to be too high-risk for open surgery performed at a major aortic center (8), or if the indication for repair is aortic rupture (9). Second, it has been formally recommended that the use of endovascular therapy in MFS patients should be limited to situations in which the stent graft can land in graft both proximally and distally (for example, to treat a focal intercostal patch aneurysm) (6). While these guidelines are a good starting point for treatment planning in patients with MFS, real-world published experiences with endovascular therapy (especially aortic endograft placement) in MFS are sparse.

The largest individual patient series addressing aortic stent grafting in MFS consists of only 16 patients (10). In all, $56 \%$ were treated successfully, but the remainder demonstrated a "primary failure" of the procedure, often requiring conversion to an open operation. Mortality was $43 \%$ in patients with primary endovascular failure, and $25 \%$ overall. Another series, encompassing 12 patients, was published by Botta et al. (11). They concluded that endovascular repair was feasible with limited intraoperative or short-term complications. However, in midterm follow up, $25 \%$ of patients developed endoleaks-and $25 \%$ demonstrated new dissection into previously unaffected aortic tissue.

The most comprehensive publication to date was performed by Pacini et al. (12). This was a systematic review of the use of stent grafting in MFS patients with type B aortic dissection (TBAD). Their search strategy identified 54 total patients across 12 previous publications. In all, $81 \%$ of the procedures involved the landing of a stent graft in the native aorta proximally. In keeping with other published works, they noted a low rate of in-hospital death (1.9\%), conversion to open surgery (3.7\%), spinal ischemia (1.9\%), and stroke (1.9\%). However, they found a remarkable rate of complications and death during follow up. Endoleaks were particularly common. The overall incidence of endoleak was $22 \%$ (18\% being type I or III that would, presumably, require reintervention). Of note, cases in which the stent grafts were landed in a graft demonstrated zero endoleaks. Stent grafting for chronic TBAD was associated with a $31 \%$ rate of endoleak, much higher than the $9 \%$ endoleak rate in acute TBAD cases. Of these patients, $16 \%$ required additional stent grafting, $18 \%$ required a subsequent open operation and $12 \%$ died during an average follow-up period of 2.5 years, with $67 \%$ of these deaths classified as being aortic related.

Many complications of aortic stent graft placement in MFS appear to result from direct injury to the intima from the stent graft, inducing a so-called "stent graft-induced new entry" (SINE) tear (2). The incidence of this was reported to be 11-fold higher than in patients without MFS. Retrograde type A dissection (RTAD) is a particular risk in patients with MFS (13).

\section{Current evidence on endovascular therapy in other connective tissue disorders}

Given that other connective tissue disorders, such as Ehlers-Danlos and Loeys-Dietz syndromes, have similar predilections for dissection, aneurysm, and rupture of the aorta and other large arteries, it is instructive to consider published series of the endovascular management in patients with these disorders. While the exact pathophysiology and histology of the diseases are different, the concerns about SINE, progressive arterial degeneration, and access site misadventures are the same, and lessons from the management patients with any of these disorders may help direct treatment in other disease states.

A recent series of 26 patients with EDS undergoing non-aortic endovascular interventions was published by Lum et al. (14). Most of these patients were young females, and $73 \%$ of the procedures catalogued were venous. In arterial cases, access was usually achieved with percutaneous puncture, and no sheaths larger than $8 \mathrm{Fr}$ were used. The majority of procedures were performed via a $5 \mathrm{Fr}$ sheath, and hemostasis was achieved with manual pressure in $69 \%$ of procedures. Fifteen percent of closures were performed 

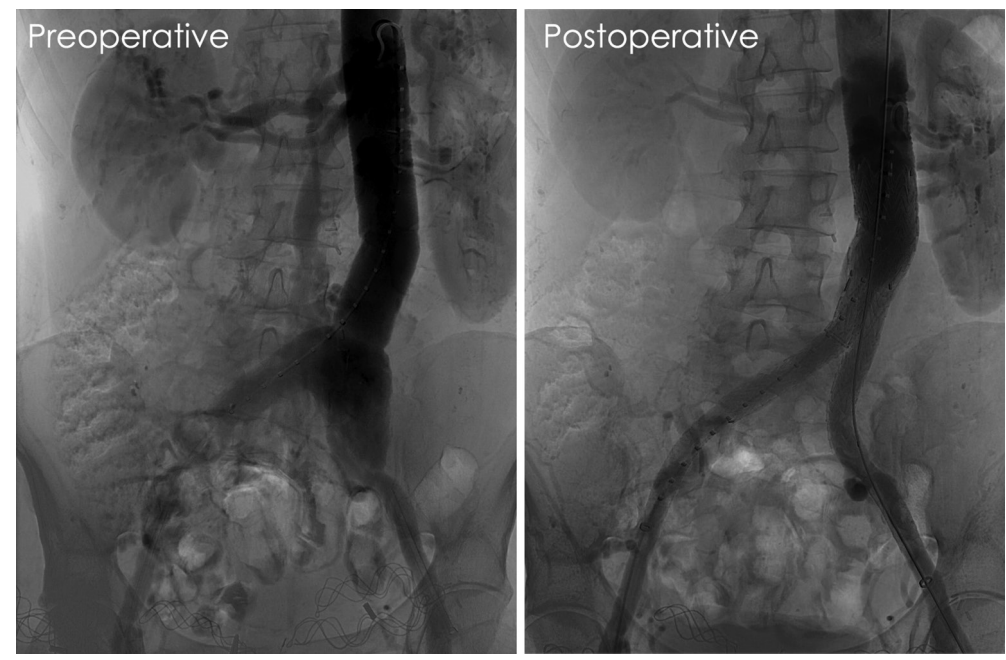

Figure 1 An endovascular aortic repair (EVAR) in a patient with a vascular connective tissue disorder. The proximal landing zone of the endovascular device is within a Dacron graft placed during an open thoracoabdominal aortic aneurysm (TAAA) repair.

with Perclose devices, and the remainder with open vessel repair after exposure prior to sheath insertion. The authors report an impressively low overall complication rate of $2 \%$. Interestingly, they found no association between procedural specifics and the development of complications. There were no access-site complications. Over a median follow up of 7.5 years, they noted a $6 \%$ total complication rate (target vessel dissection, type $\mathrm{B}$ dissection, and death) with only 1 clearly related to the endovascular procedure itself.

Important conclusions can be drawn from this data. First, with attention to meticulous technique, percutaneous access and hemostasis can be performed with very low risk in patients with MFS. Second, the drawbacks of endovascular treatments are realized over midterm follow up, and predominantly manifest as endoleaks, though retrograde dissection is a significant concern. A proximal in-graft landing zone should be sought whenever feasible.

\section{Our perspective on the endovascular therapy in MFS}

In determining the best course of action for treating patients with a vascular manifestation of MFS, it is useful to consider the advice that has traditionally guided the open repair of proximal aortic issues in these patients: "For patients with acute manifestations... the goal of surgery is preservation of life, and the most expedient repair... as opposed to the more erudite repairs offered in elective scenarios" (3). In keeping with this philosophy, we believe there are several noteworthy scenarios that may justify endovascular therapy in MFS and other similar connective tissue disordersscenarios in which an expedient repair is more beneficial than the "gold standard." These include: (I) treatment of rupture; (II) exclusion of patch aneurysms; (III) treatment of certain anatomical areas, such as the intercostal or vertebral arteries, in which open exposure is prohibitively morbid; and (IV) elective repairs in patients who desire a minimally invasive approach and/or are high-risk for open surgery, in which the stent graft can be landed within a surgically placed Dacron graft, especially proximally (Figure 1).

In order to minimize complications during endovascular treatments, certain techniques and strategies can be employed. Ultrasound guidance during percutaneous access is mandatory. Occasionally, the fragile arteries in these patients may be dissected or the anterior wall may be obliterated by moderate or large sheath placement, so direct midline vessel puncture in a healthy segment of common femoral artery is crucial to reduce this risk. If open vessel exposure is required, several strategies are beneficial. Manual pressure can be effective in controlling bleeding encountered during surgical exposure and may be preferred to clamp placement or suture ligation. A padded clamp should be used if clamping is required. If suture repair of a vessel is needed, we tend to use a two-layer approach involving both continuous and interrupted layers. First, a running suture is used to approximate all layers of the arterial wall with carefully placed but generously sized bites. Next, we place interrupted U-stitches, pledgeted on both sides of the suture 
line, with the needle entering from "outside to inside" on the proximal aspect, and "inside to outside" on the distal aspect, to avoid lifting a dissection flap that could impede flow distally. This differs from the approach described by other authors involving a circumferential "collar" of felt that is used to reinforce the initial vessel anastomotic suture line. Importantly, our technique avoids the need for circumferential vessel exposure which, if pursued, could lead to injury to neighboring veins or a posterior anastomotic defect that would be difficult to control. Finally, the induction of hypotension during clamp removal/flow restoration is critical. Nuisance needle-hole bleeding can easily convert to localized pseudoaneurysms or anastomotic dehiscence if the fragile vessel layers delaminate. On occasion, we have had to reapply clamps and lower the systolic blood pressure multiple times in order to find an appropriate threshold that the anastomosis can tolerate while maintaining a reasonable perfusion pressure to the rest of the body. Anti-impulse therapy with beta blockade may be required to maintain hypotension in an intensive care unit for the first day or two postoperatively.

After endovascular intervention, patients with MFS are seen in our outpatient clinic with CTA surveillance at 1, 6, and 12 months, and then yearly thereafter.

\section{Conclusions}

Endovascular therapy for MFS can be successful. However, it is associated with a unique set of risks during midterm follow up. It is appropriate in certain situations, as outlined above. As with any vascular operation, preoperative planning and intraoperative judgment are crucial to ensuring a good outcome for the patient.

\section{Acknowledgements}

The authors acknowledge Dr. Hazim J. Safi for the help in this study.

\section{Footnote}

Conflicts of Interest: Dr. Azizzadeh is a consultant for WL Gore and Medtronic; Dr. Tjaden has no conflicts of interest to declare.

\section{References}

1. Cikrit DF, Miles JH, Silver D. Spontaneous arterial perforation: the Ehlers-Danlos specter. J Vasc Surg 1987;5:248-55.

2. Dong $Z, F u W$, Wang $Y$, et al. Stent graft-induced new entry after endovascular repair for Stanford type B aortic dissection. J Vasc Surg 2010;52:1450-7.

3. Black JH III. Aneurysms Caused by Connective Tissue Abnormalities. In: Cronenwett JL, Johnston KW. editors. Rutherford's Vascular Surgery. 8th edition. Philadelphia, PA: Elsevier/Saunders, 2014:2248-67.e3.

4. Glebova NO, Hicks CW, Alam R, et al. Technical aspects of branched graft aortic reconstruction in patients with connective tissue disorders. J Vasc Surg 2016;64:520-5.

5. Doyle A, Doyle JJ, Dietz HC. Chapter 702: Marfan Syndrome. In: Kliegman RM, Stanton BM, St. Geme J, et al. editors. Nelson Textbook of Pediatrics 20th edition, Elsevier, 2016: 3384-9.e1.

6. Milewicz DM, Dietz HC, Miller DC. Treatment of aortic disease in patients with Marfan syndrome. Circulation 2005;111:e150-7.

7. Dardik A, Perler BA, Roseborough GS, et al. Aneurysmal expansion of the visceral patch after thoracoabdominal aortic replacement: an argument for limiting patch size? J Vasc Surg 2001;34:405-9; discussion 410.

8. Svensson LG, Kouchoukos NT, Miller DC, et al. Expert consensus document on the treatment of descending thoracic aortic disease using endovascular stent-grafts. Ann Thorac Surg 2008;85:S1-41.

9. Hiratzka LF, Bakris GL, Beckman JA, et al. 2010 ACCF/AHA/AATS/ACR/ASA/SCA/SCAI/SIR/STS/ SVM guidelines for the diagnosis and management of patients with Thoracic Aortic Disease: a report of the American College of Cardiology Foundation/American Heart Association Task Force on Practice Guidelines, American Association for Thoracic Surgery, American College of Radiology, American Stroke Association, Society of Cardiovascular Anesthesiologists, Society for Cardiovascular Angiography and Interventions, Society of Interventional Radiology, Society of Thoracic Surgeons, and Society for Vascular Medicine. Circulation 2010;121:e266-369.

10. Waterman AL, Feezor RJ, Lee WA, et al. Endovascular treatment of acute and chronic aortic pathology in patients with Marfan syndrome. J Vasc Surg 2012;55:1234-40; disucssion 1240-1.

11. Botta L, Russo V, La Palombara C, et al. Stent graft repair of descending aortic dissection in patients with Marfan syndrome: an effective alternative to open reoperation? J Thorac Cardiovasc Surg 2009;138:1108-14. 
12. Pacini D, Parolari A, Berretta P, et al. Endovascular treatment for type B dissection in Marfan syndrome: is it worthwhile? Ann Thorac Surg 2013;95:737-49.

13. Dong ZH, Fu WG, Wang YQ, et al. Retrograde type A aortic dissection after endovascular stent graft placement for treatment of type B dissection. Circulation
2009;119:735-41.

14. Lum YW, Brooke BS, Arnaoutakis GJ, et al. Endovascular procedures in patients with Ehlers-Danlos syndrome: a review of clinical outcomes and iatrogenic complications. Ann Vasc Surg 2012;26:25-33.
Cite this article as: Tjaden B Jr, Azizzadeh A. Endovascular therapy in Marfan syndrome: PRO. Ann Cardiothorac Surg 2017;6(6):672-676. doi: 10.21037/acs.2017.10.06 